\title{
From Art to Part: A Simultaneous Geometry and Decoration Workflow
}

\author{
Yajie Jiang \\ jiangy3@mcmaster.ca
}

\author{
Simon El Sahi \\ elsahis@mcmaster.ca
}

\author{
Allan D. Spence \\ adspence@mcmaster.ca
}

\begin{abstract}
Reproduction of hand crafted $3 D$ shape geometry is an important industrial concept design step employed for items ranging in size and function. Adding surface art work would extend concept design to include essential product information.

This paper describes an equipment and workflow implementation for simultaneously reproducing both $3 D$ shapes and art work. The handcrafted shape is laser digitized to obtain the XYZ geometry coordinates plus the surface gray level. The resulting point cloud is processed and converted to an STL file for plastic rapid prototyping, while the surface gray level information is resampled to fit the smoothed surface and match the reproduction ink jet printing system resolution. Developed software automatically calculates the dot pattern to reproduce the art work onto the correct prototyped surface location. An actual sample produced using the system is shown.
\end{abstract}

\section{Introduction}

The high incidence of product failure in the marketplace pushes companies to focus on improving the product quality at development stages [1]. However, product design and prototyping usually require many modifications, reruns, and reproductions of decorated hand crafted models. In this paper a workflow for 3D decorated shapes reproduction is presented. This workflow utilizes laser digitizing, rapid prototyping, applications of CAD tools and 3D ink-jet printing. It is easy to implement, and its repeatability and functionality provide meaningful help to design engineers.

To implement the workflow the 3D shape is scanned and point cloud data (PCD) with its corresponding gray level is collected, and then converted to a CAD model. The workflow involves laser digitizing, CAD, B-spline curves and surfaces, rapid prototyping, and oriented ink jet printing.

\section{System Architecture}

The art-to-part reproduction system is composed of two primary sub systems. To capture the geometric surface data and its corresponding gray level data, a 3D laser digitizing system is used as the input system. For output, a fused deposition modeling (FDM) rapid prototyping machine is used to create the part geometry and an oriented 3D ink-jet printing system is used to reproduce the artwork.

\subsection{CMM-Laser Digitizing System}

The laser digitizer system, shown in Fig. 11, is based on a Hymarc 45C/SC2 laser digitizer [2] mounted on a bridge style coordinate measuring machine CMM. A computer workstation, connected to the digitizer controller and the OTC5000 CMM controller, is used to manage the CMM position and integrate it with the collected point cloud data (PCD) and gray level information. The laser digitizer captures the surface 3D coordinates with their intensities employing the principle of active triangulation synchronized scanning. A $3.3 \mathrm{~Hz}$ laser object surface sweeping frequency along with the CMM feeding speed of $0.33 \mathrm{~mm} / \mathrm{sec}$ generates a point cloud with 10 point/mm density.

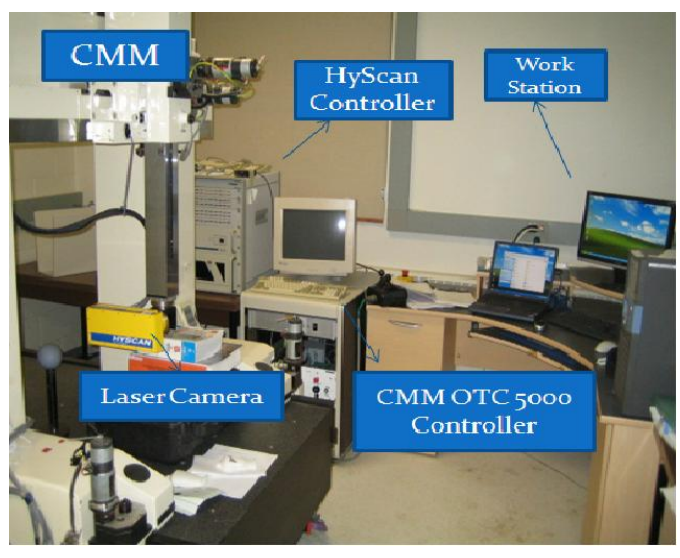

Figure 1: CMM-Hymarc laser digitizing system 


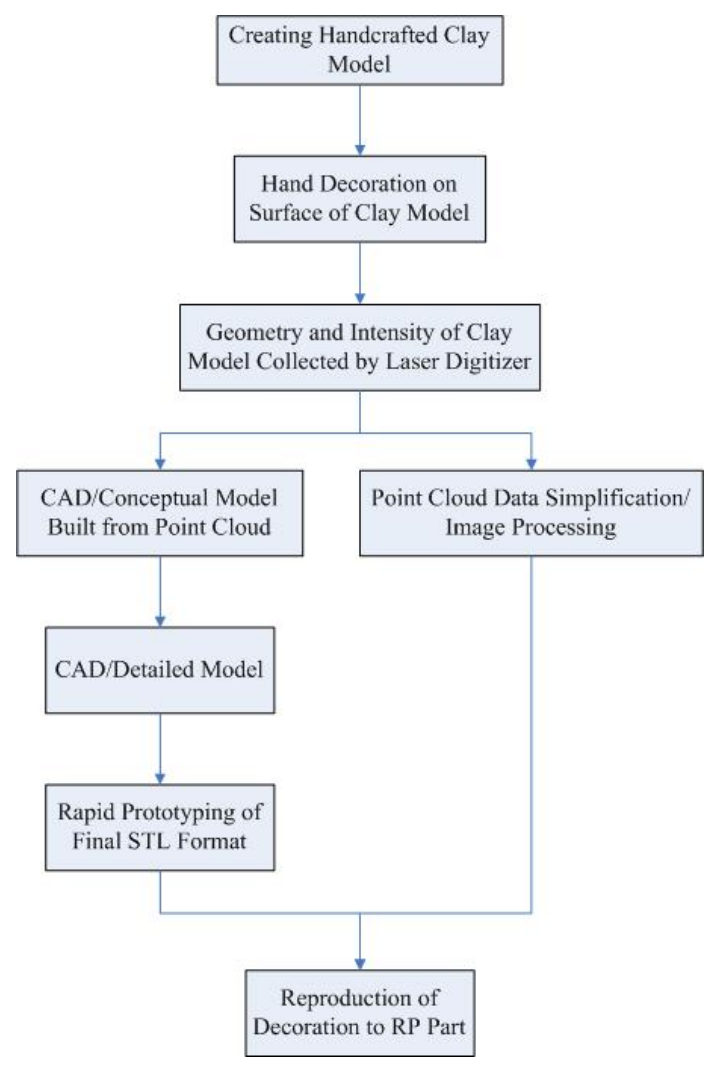

Figure 2: General workflow

\subsection{Rapid Prototyping Machine}

Rapid prototypes are created using the Dimension BST Fused Deposition Modelling machine [3].

\subsection{Oriented 3D Ink-jet Printer}

An oriented 3D Ink-jet printer system was set-up primarily to print on non-porous 3D surfaces. The system (Fig. 7) consists of a coordinate measuring machine (CMM), orientable probe head, an ink-jet controller, and a print head. A washable ink adhesive coating [4] reduces costs by permitting cleaning and reuse of the rapid prototype part during decoration trials.

\section{Workflow Implementation}

A three-staged product development workflow employing CAD modeling tools is shown in Fig. 2. In the first stage, point cloud data (PCD) is collected from the hand crafted model. In the second stage the data is processed to create a CAD geometry model, a binary image of the artwork, and a rapid prototyped model. In the final stage the model is decorated with processed artwork.

\subsection{Hand crafted Model PCD Collection}

The hand crafted model is made from Crayola air-dry clay [5] and pre-coated with inkAID white matte. Half of the handcraft model is built initially to allow it to lie horizontally on the work jig (Fig. 3a). Artwork is added to its relatively flat surface to improve laser digitizing and ink-jet printing processes performance. Assembling the hand crafted model is achieved by applying glue to the mating surfaces. The digitized features include the geometry of the prototype body surface with the corresponding intensity and some portions of interest on the work jig such as the top surfaces of jig plate and the three stand-offs (Fig. 3b), which are used to locate relevant coordinate system datums.

\subsection{PCD Conversion to STL Format}

Software preparation of the conceptual model is accomplished using AliasStudio, Autodesk Inventor [6], and EDS Imageware [7].

A detailed workflow to build a sample product conceptual model is shown in Fig. 4 First, the geometric information of main body edges are extracted and sampled from original PCD. A uniform parametrization least squares algorithm [8] computes a small set of BSpline control points to use in constructing the surface edges in Autodesk Inventor. These generated surface curves are then compared to original PCD geometry for modification and body surface construction in AliasStudio and Imageware. The verification is attained by aligning ordered datum plates of the conceptual and original models. The construction various stages are depicted in Fig. 5 where the verification results in a conformity error of less than $1 \mathrm{~mm}$ in the image areas of the surface. Good image reproduction can be easily attained within the resulting error margins. To produce the conceptual model, its surface is thickened in Inventor to meet the rapid prototyping machine requirements.

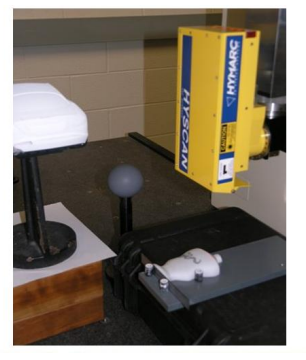

(a) Prototype digitization

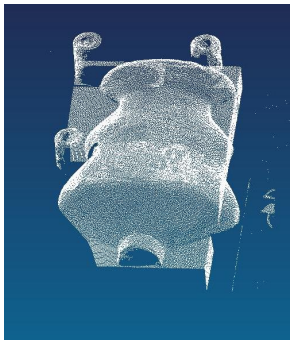

(b) Result PCD
Figure 3: PCD collection from prototype 


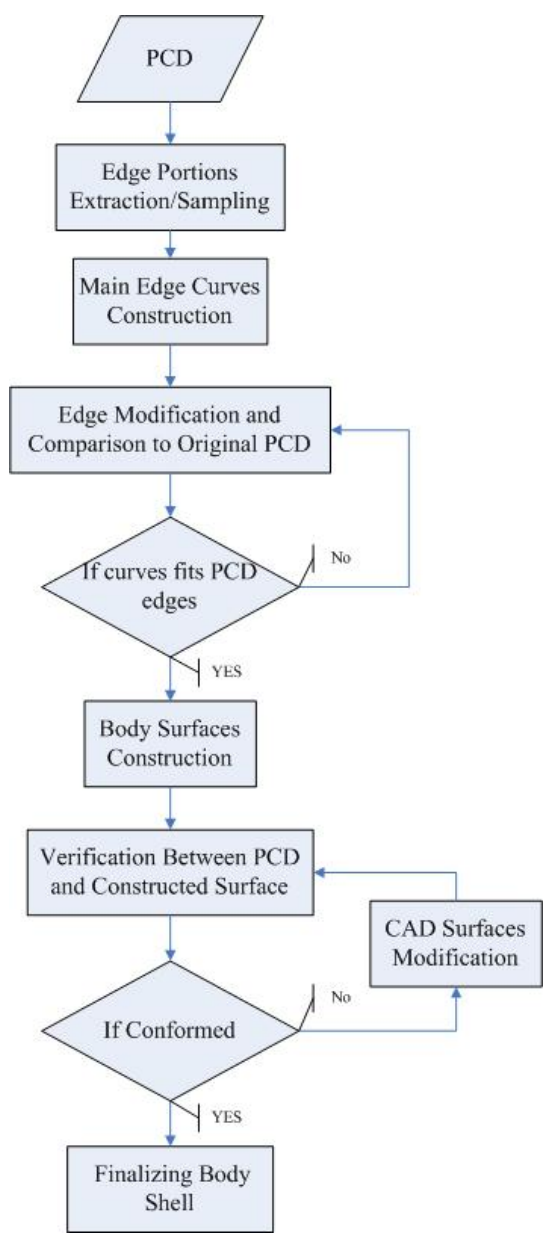

Figure 4: Conceptual model construction workflow

\subsection{Image Processing}

The objective of image processing is to create a 2-D image from original PCD where the image pixel density is compatible with the oriented 3D ink-jet printer. For this task the original data is orthogonally sorted in the xy domain, and then re-sampled from a 254 DPI (10 points $/ \mathrm{mm}$ ) resolution to $96 \mathrm{DPI}$. The resulting $2 \mathrm{D}$ gray and binary images are shown in Fig. 6, and the data simplification steps are described as follows:

1. The image box size required for printing is estimated to be $1 \times \mathrm{h}$ where 1 and $\mathrm{h}$ are the length and height of the image in the xy plane. The extracted lxh sized image PCD is orthogonally sliced into pixel grids in the $\mathrm{xy}$ plane with a constant square size of $\mathrm{g} \times \mathrm{g}$, where $\mathrm{g}$ is equal to $1 / 96$ inch. 2 . The point cloud data is simplified to create a cell array with respect to the grid map, resulting in all the points in the same grid being dragged to its upper left corner. The corresponding $\mathrm{z}$ and intensity $\mathrm{I}$ are calculated by averaging all values of $\mathrm{z}$ and $\mathrm{I}$ in this grid area from the original PCD.
3. A gray image bitmap array is constructed by extracting all intensity values and realigning them into the proper positions with respect to the simplified PCD array. Next, the gray image is converted into a binary one by employing Otsu's Threshold Algorithm for image binarization [9].

\subsection{Image Oriented Ink-Jet Printing}

The final step of the artwork reproduction is oriented 3D ink-jet printing (Fig. 7). This process starts by analyzing the tangent values of each edge profile corresponding with the image location every 1/96th inches to determine the orientation of the artwork local coordinate system (LCS) on the CMM table. By touch probing the jig fixture used during the laser digitizing a computed transformation matrix is used to convert the LCS values into machine coordinate system (MCS) values. The prototype built using the surface smoothed data is then coated with InkAID Clear Glass Type II to enhance ink adhesiveness to the part and allow part washing for reuse.

By using the image sectioning method described in El Sahi et al. [10], the most curved surface direction is subdivided into planar sections corresponding to the 7.5 degree head index increment. In the less curved direction, specially developed software determines the printing paths, and converts the image sections into dot pat-

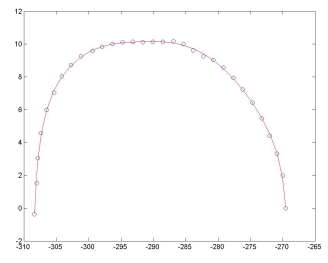

(a) Upper edge profile

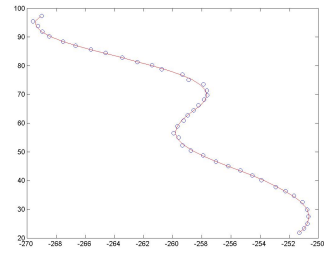

(b) Side edge profile

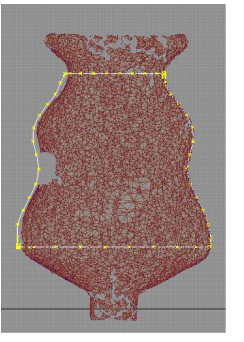

(c) CAD main edges modification

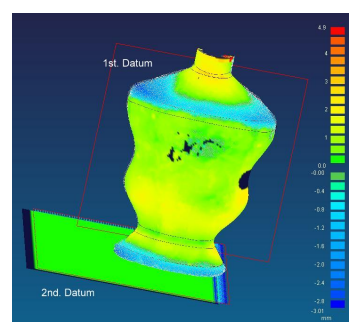

(d) CAD surface verification
Figure 5: Conceptual model implementation results 


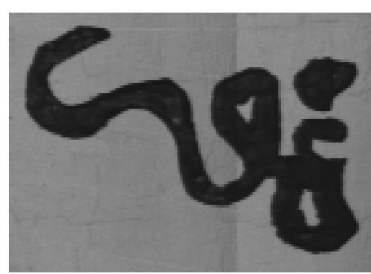

(a) Gray Level Image

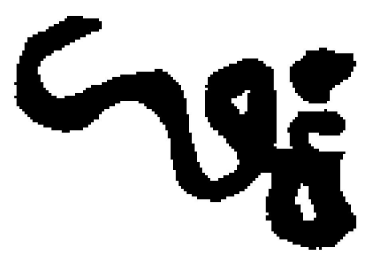

(b) Binary Image

Figure 6: Image processing results

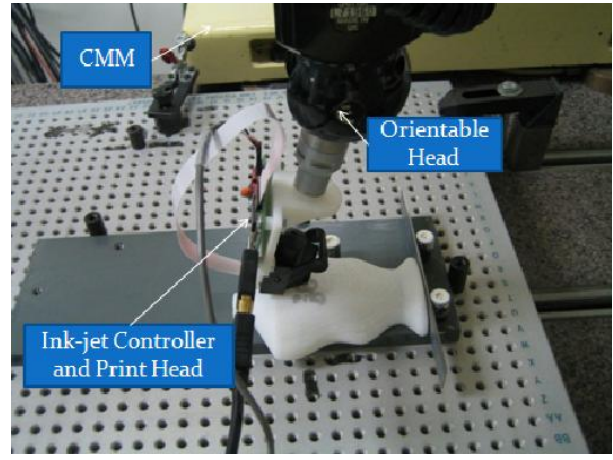

Figure 7: Oriented 3D ink-jet printing

terns which are sent through a serial connection to the printer microcontroller. The printer microcontroller automatically sprays the next row of dots each time it receives a synchronizing TTL signal that is transmitted by the CMM motion controller every 1/96th inch of travel.

\section{Experimental Results And Verification}

As an illustration case, an artistic Chinese character has been painted on a handcraft clay model which was scanned, rapid prototyped and decorated using an oriented 3D ink-jet printer. Fig. 8 shows the original handcraft along with the CAD replica stages with no visible image dislocation or deformation.

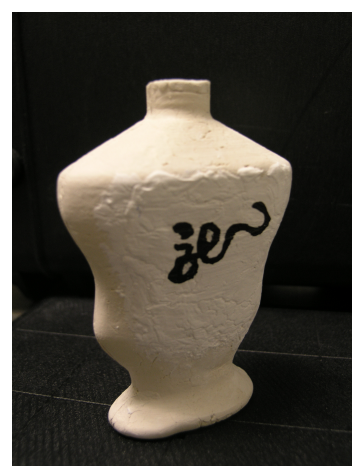

(a) Handcrafted prototype

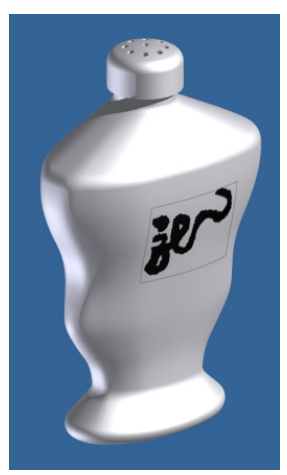

(b) CAD model

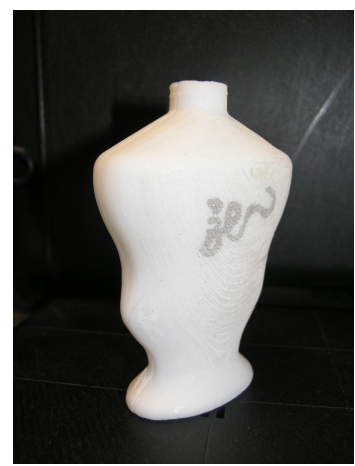

(c) Reproduced RP model

Figure 8: Experimental results

\section{Summary}

This paper has demonstrated a product development workflow to convert decorated handcraft concept models into reproducible and functional prototypes. Reverse engineering of a handcraft model with surface artwork was captured with laser digitization, visualized and optimized through CAD and surface modellers, and built using FDM rapid prototyping technology. By using oriented 3D ink-jet printing, the resulting binary image from processing the gray level data associated with the surface points was reproduced on the surface with a resolution of 96 DPI and a positional error of $1 \mathrm{~mm}$. 
The introduction of this workflow implementation allows artists and designers to create without the constraints of functionality or repeatability of the prototype.

\section{Acknowledgments}

This work was financially supported by the Natural Sciences and Engineering Research Council of Canada.

\section{References}

[1] G. F. Smith. Quality Problem Solving. ASQ Quality Press, Milwaukee, 1998.

[2] Ltd. Hymarc 3D Vision System. Hyscan 3-d Laser Digitizing System- Operator's Manual. Nepean, ON, Canada. Available from: www. hymarc.com.

[3] Stratasys, Inc. Eden Prairie, MN, USA. http://www. dimensionprinting.com.

[4] Ontario Specialty Coatings Corp.: inkAID, Watertown, NY, USA. http: / / www. inkaid1. com.
[5] Crayola: Lindsay, ON, Canada. http: //www.crayola.com.

[6] Autodesk, Inc.: Inventor, San Rafael, CA, USA. http:// www.autodesk.com.

[7] Electric Data Systems Corporation: Imageware 11, Plano, TX, USA. http: / /www. eds . com.

[8] L. Piegl and W. Tiller. The NURBS book (2nd ed.). Springer-Verlag New York, Inc., New York, NY, USA, 1997.

[9] N. Otsu. A threshold selection method from gray-level histograms. IEEE Transactions on Systems, Man and Cybernetics, 9(1):62-66, January 1979.

[10] S. El Sahi, Y. Jiang, and A.D. Spence. Oriented 3-d inkjet print. Computer-Aided Design and Applications, 3(14):399-406, 2009. 